\title{
Principles of calculating the dynamical response of misaligned complex resonant optical interferometers
}

\author{
Daniel Sigg \\ LIGO Hanford Observatory, P.O. Box 1970 S9-02, Richland, Washington 99352 \\ Nergis Mavalvala \\ LIGO Project, California Institute of Technology, Pasadena, California 91125
}

\begin{abstract}
Received October 20, 1999; revised manuscript received May 10, 2000; accepted May 12, 2000
In the long-baseline laser interferometers for measuring gravitational waves that are now under construction, understanding the dynamical response to small distortions such as angular alignment fluctuations presents a unique challenge. These interferometers comprise multiple coupled optical resonators with light storage times approaching $100 \mathrm{~m}$. We present a basic formalism to calculate the frequency dependence of periodic variations in angular alignment and longitudinal displacement of the resonator mirrors. The electromagnetic field is decomposed into a superposition of higher-order spatial modes, Fourier frequency components, and polarization states. Alignment fluctuations and length variations of free-space propagation are represented by matrix operators that act on the multicomponent state vectors of the field. (c) 2000 Optical Society of America [S0740-3232(00)01509-X]
\end{abstract}

OCIS codes: $120.3180,120.2230,030.4070,220.1140$.

\section{INTRODUCTION}

A new generation of ground-based laser interferometric gravitational-wave detectors with arm lengths of up to 4 $\mathrm{km}$ is currently under construction. ${ }^{1-4}$ Their sensitivity to relative length changes is expected to be $\Delta L / L$ $\sim 10^{-21} \mathrm{rms}$ integrated over a $1-\mathrm{kHz}$ bandwidth centered in the region of minimum noise. To achieve such high sensitivity, these systems implement complicated variations of a Michelson interferometer with multiple coupled optical resonators. The long baselines and the coupled cavities of the optical resonators yield light storage times on the order of $0.5-100 \mathrm{~ms}$. To maintain resonance conditions, the interferometer lengths must be tuned to within a small fraction of the cavity linewidth. To further ensure maximum sensitivity to gravitational waves, the interferometer mirrors must be held in precise angular alignment with respect to the incoming laser beam. ${ }^{5}$ To implement a control system to keep the interferometer lengths closely tuned to resonance and to accurately infer the sensitivity to gravitational waves, the frequency response of the interferometer to changes in resonant lengths must be known. Similarly, understanding the interferometer response to angular misalignment is necessary to enable an alignment control system.

In the past, two separate but mathematically similar formalisms have been independently developed: One describes the dynamical behavior of slightly off-resonance interferometer fields, ${ }^{6,7}$ and the other is useful for calculation of the effects of angular misalignment. ${ }^{8-12}$ Both approaches are based on decomposing the laser field into a multicomponent vector. In the dynamical response formalism, the vector components represent different fre- quencies of the laser light, while in the angular misalignment formalism they represent different spatial modes of the field. In both approaches beam propagation and distortion effects are expressed as operators acting on these field vectors, making it possible to simulate complex resonant optical systems by evaluating relatively straightforward matrix equations.

In this paper we combine the two formalisms by defining a vector space that includes both the frequency and the spatial mode components of the field. For completeness we include the light polarization explicitly. Using a triangular ring cavity as a simple example, we derive the operators for propagation and reflection when both the resonant lengths and the mirror angles are dithered at a given frequency (Section 2). We then calculate the frequency response of the optical system in conjunction with a heterodyne detection scheme (Section 3) and in the presence of noise terms in the input light (Section 4). Finally, we provide a recipe for building more complex optical systems such as coupled Fabry-Perot cavities in the arms of a Michelson interferometer (Section 5). We emphasize the generality of the model as an analytic tool to investigate the frequency dependence of distortion effects in any complex optical system-in particular, coupled cavity systems.

\section{BASIC FORMALISM}

It is useful to first study a relatively simple optical system, a triangular ring cavity, shown in Fig. 1. The laser field inside the cavity, $E_{\text {cav }}$, can be expressed as the sum 


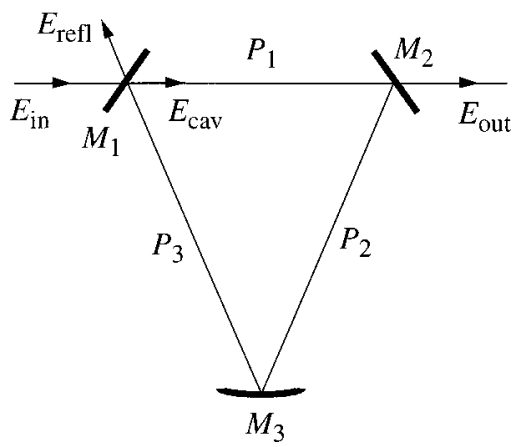

Fig. 1. Triangular Fabry-Perot cavity.

of the input field $E_{\text {in }}$ and the field circulating in the cavity (which is due to the previous round trip), $E_{\text {cav }}^{\prime}$ :

$$
E_{\text {cav }}=G_{\mathrm{rt}} E_{\text {cav }}^{\prime}+T_{1} E_{\text {in }},
$$

where $G_{\mathrm{rt}}$ denotes an operator that propagates the laser field by one round trip and where $T_{1}$ describes the transmission through the input mirror $M_{1}$. In the steady state, the cavity resonance condition is satisfied when $E_{\text {cav }}=E_{\text {cav }}^{\prime}$. It is important to recognize that this condition describes not only the static case, but also situations with periodic variations by using field vectors consisting of multiple frequency components.

In the paraxial approximation, the electromagnetic field vector of a light beam can be expressed as a superposition of orthonormal Gaussian modes. ${ }^{13}$ If the time dependence of the field is periodic at frequency $f$, one can write the electric field in terms of Fourier components:

$$
\begin{aligned}
\mathbf{E}(x, y, z, t)= & \sum_{m n} \sum_{r} \sum_{p} a_{m n r p} \exp \left[i\left(\omega_{0}+r \omega\right) t\right] \\
& \times U_{m n}(x, y, z) \boldsymbol{\epsilon}_{p},
\end{aligned}
$$

where $\omega_{0}$ is the angular frequency of the laser light, $\omega$ $=2 \pi f$ is the fundamental angular frequency of the variation, and $\epsilon_{1}$ and $\boldsymbol{\epsilon}_{2}$ are the two transverse polarizations in $x$ and $y$, respectively. Naturally, the $U_{m n}(x, y, z)$ are chosen to represent the eigenmodes of an optical resonator.

For instance, a Hermite-Gaussian beam ${ }^{13}$ can be described by

$$
\begin{aligned}
U_{m}(x, z)= & \left(\frac{2}{\pi}\right)^{1 / 4}\left[\frac{1}{2^{m} m ! w(z)}\right]^{1 / 2} H_{m}\left[\frac{\sqrt{2} x}{w(z)}\right] \\
& \times \exp \left(-x^{2}\left\{\frac{1}{[w(z)]^{2}}+\frac{i k_{0}}{2 R(z)}\right\}\right) \\
& \times \exp \left[i\left(m+\frac{1}{2}\right) \eta(z)\right],
\end{aligned}
$$

where the $z$ axis points in the beam propagation direction and where $\eta(z), w(z)$, and $R(z)$ are the mode-dependent Gouy phase shift, the spot size, and the curvature of the phase front at position $z$, respectively, given by

$$
\eta(z)=\tan ^{-1}\left(\frac{z}{z_{0}}\right), \quad w(z)=w_{0}\left[1+\left(\frac{z}{z_{0}}\right)^{2}\right]^{1 / 2},
$$

$$
R(z)=z+\frac{z_{0}^{2}}{z}
$$

and the Rayleigh length $z_{0}$ is given by $z_{0}=\pi w_{0}^{2} / \lambda$, with $w_{0}$ as the waist size. $H_{m}(x)$ is the Hermite polynomial of order $m$. In two dimensions the Hermite-Gaussian modes are given by

$$
U_{m n}(x, y, z)=U_{m}(x, z) U_{n}(y, z) .
$$

In its most general form, the quantity $a_{m n r p}$ of Eq. (2) represents a tensor of rank 4 . The indices $m, n$ refer to the spatial mode component, the index $r$ refers to the Fourier frequency component, and $p$ refers to the polarization state of the electric field. Since in most practical applications an operator acting on $a_{m n r p}$ will contract over all four indices simultaneously-i.e., it will sum over all spatial modes, frequency components, and polarization states-one can map $a_{m n r p}$ into a one-dimensional vector space. Assuming that only a finite number of frequency components, $n_{f}$, are considered, such that $-\left(n_{f}-1\right) / 2$ $\leqslant r \leqslant\left(n_{f}-1\right) / 2$, one can write, for example, $a_{m n r p}$ $\rightarrow a_{i}$ :

$$
i=2 n_{f}\left[\frac{(m+n)(m+n+1)}{2}+n\right]+a(r)+p,
$$

with

$$
a(r)=\left\{\begin{array}{ll}
r, & r \geqslant 0 \\
r+n_{f}, & r<0
\end{array} .\right.
$$

This convention allows us to use standard linear algebra packages to perform numerical calculations; Mathematica $^{\mathrm{TM}}$ was used to evaluate the examples in this paper. Our goal is to derive matrix operators acting on this vector space, which account for free-space propagation and reflection from a mirror. We can then calculate the reflected and transmitted fields, $E_{\text {refl }}$ and $E_{\text {out }}$, respectively, by solving simple matrix equations.

Using the operators $P$ and $M$ in Eq. (1) for propagation and reflection from a mirror surface, we can write the round-trip operator as

$$
G_{\mathrm{rt}}=M_{1} R P_{3} M_{3} P_{2} M_{2} P_{1} .
$$

We follow the convention that the $z$ axis is always defined along the beam direction, and the $x$ and $y$ axes are transverse and orthogonal. In general, the $x$ and $y$ axes preserve the orientation of the beam image after reflection from a mirror surface; i.e., the coordinate system alternates between right handed and left handed with every reflection. However, the $x$ and $y$ axes must align again after one round trip of the ring cavity. In general, both a rotation of the beam (nonplanar configuration) and a parity operation (odd number of reflections) may occur (see Appendix A1); this is represented by the operator $R$ in Eq. (8). For a triangular cavity, however, only a parity operation that flips the beam about the vertical axis is necessary. To form a stable cavity, at least one of the mirrors must be curved. To neglect astigmatic effects in a triangular cavity, we assume that the length between $M_{1}$ and $M_{2}$ is much shorter than the two other mirror separations (see Fig. 1).

Propagation in free space along the $z$ axis adds a term that is due to the Gouy phase shift, $\exp [i(m+n+1) \eta]$, 
which depends only on the spatial mode order and does not affect the polarization. Since a change of length affects neither the modal decomposition nor the polarization of the field, one can write

$$
P_{m n r p, k l s q}=P_{m n, k l}^{M} \otimes P_{r s}^{F} \otimes P_{p q}^{P},
$$

where $P^{M}, P^{F}$, and $P^{P}$ are operators acting on the modal space, the frequency space, and the polarization states, respectively. The outer product $\otimes$ is used to combine operators that act on subspaces independently. For instance, in the above equation the modal operator $P^{M}$ is applied to all frequency components and polarization states identically. Following Ref. 12, one can write beam propagation as

$$
P_{m n, k l}^{M}(\eta)=\delta_{m k} \delta_{n l} \exp [i(m+n+1) \eta],
$$

with $\eta=\eta\left(z_{2}\right)-\eta\left(z_{1}\right)$. The propagator $P_{p q}^{P}$ accounting for polarization effects is unity. Assuming that the distance $l(t)=z_{2}-z_{1}+\Delta l(t)$ is modulated by a small periodic signal $\Delta l(t)$ at frequency $f$, one can write the Fourier series as

$$
l(t)=\sum_{\rho} l_{\rho} \exp (i \rho \omega t),
$$

with $l_{0}=z_{2}-z_{1}$. The propagator $P_{r s}^{F}$ then becomes

$$
\begin{aligned}
P_{r s}^{F}(l)= & \left\langle r\left|\exp \left[-i \sum_{\rho}\left(k_{0}+\frac{\rho \omega}{c}\right) l_{\rho} \exp (i \rho \omega t)\right]\right| s\right\rangle \\
= & \exp \left[-i k_{0}\left(z_{2}-z_{1}\right)\right] \\
& \times\left\langle r\left|\exp \left[-i \sum_{\alpha \beta}|\alpha\rangle L_{\alpha \beta}\langle\beta|\right]\right| s\right\rangle
\end{aligned}
$$

where $k_{0}$ denotes the wave number of the laser light, the bra-ket product is defined as the integration in time over a full period, and $\langle r|$ and $|s\rangle$ are the Fourier basis vectors $\exp (-i r \omega t)$ and $\exp (i s \omega t)$, respectively. If we neglect terms of order $l_{\rho} \omega / c \quad(\rho \neq 0)$ and use Eq. (11), $L_{\alpha \beta}$ in the exponent of Eq. (12) reduces to

$$
L_{\alpha \beta} \simeq \alpha \frac{\omega}{c}\left(z_{2}-z_{1}\right) \delta_{\alpha \beta}+k_{0} \sum_{\rho \neq 0} l_{\rho} \delta_{\alpha, \beta+\rho} .
$$

Similarly, one can derive the mirror operator $M_{m n r p, k l s q}$. Again, the polarization is an independent degree of freedom and can be treated separately. One can write the reflection from a mirror surface as

$$
\begin{aligned}
M_{m n r p, k l s q}= & M_{m n r, k l s}^{M F} \otimes M_{p q}^{P}, \\
M_{m n r, k l s}^{M F}= & \langle m n r| \exp \left[-2 i \sum_{\alpha \beta \rho, \gamma \delta \sigma}|\alpha \beta \rho\rangle T_{\alpha \beta, \gamma \delta}^{M}\right. \\
\otimes & \left.T_{\rho \sigma}^{F}\langle\gamma \delta \sigma|\right]|k l s\rangle,
\end{aligned}
$$

where the modal distortion operator $T^{M}$ is assumed to affect all frequency components the same way. The light frequency is on the order of $10^{14} \mathrm{~Hz}$, while physical disturbances of the mirrors and therefore the frequencies of modal distortions are typically in the audio band. Most reflective surfaces do not distinguish such small frequency variations of the laser light. The case where the mirror operator in Eq. (14) describes alignment fluctuations is, however, of special interest. If the mirror is dithered in angle about the $y$ axis by $\theta(t)$ $=\Sigma_{\tau} \theta_{\tau} \exp (i \tau \omega t)$, one obtains the following in the Hermite-Gaussian basis ${ }^{12}$ :

$$
\begin{aligned}
T_{\alpha \beta, \gamma \delta}^{M} & =\frac{1}{\sqrt{2}} \delta_{\beta \delta}\left(\sqrt{\gamma} \delta_{\alpha, \gamma-1}+\sqrt{\alpha} \delta_{\alpha, \gamma+1}\right), \\
T_{\rho \sigma}^{F} & =\sqrt{2} \sum_{\tau} \Theta_{\tau} \delta_{\rho, \sigma+\tau},
\end{aligned}
$$

where $\Theta_{\tau}=\theta_{\tau} \pi w(z) / \lambda$ denotes the normalized misalignment angle. If wave-front distortion effects are neglected when passing through a mirror substrate, the transmission operator becomes

$$
T=\mathbf{1}^{M} \otimes \mathbf{1}^{F} \otimes T^{P},
$$

with $\mathbf{1}$ as the unity operator. If the reflectivity is independent of polarization, the corresponding operator $M^{P}$ is just the amplitude reflectivity $r$ times the unity operator. Similarly, the transmission operator $T^{P}$ is described by the amplitude transmission coefficient only; i.e.,

$$
M_{p q}^{P}=r \delta_{p q}, \quad T_{p q}^{P}=i t \delta_{p q}
$$

(the general case is treated in Appendix A2). The convention of using an imaginary transmission coefficient makes the transmission operator $T$ the same in both directions, and it makes the distortion operator $M^{\dagger}$, which describes the reflection from the opposite direction (rear side of mirror coating), the complex-conjugated transpose of the matrix that describes reflection from the front surface (assuming that the coating is perfect).

\section{DETECTION SCHEME}

A photodetector at position $z$ measures the light power integrated over its surface area $\Omega$, i.e.,

$$
P_{\text {det }}=\frac{\epsilon_{0} c}{2} \iint_{\Omega} \mathrm{d} x \mathrm{~d} y \mathbf{E}^{*}(x, y, z) \cdot \mathbf{E}(x, y, z),
$$

where $\epsilon_{0}$ and $c$ are the free-space permittivity and the speed of light, respectively. The shape of the photodetector, including segmentation in the case of a quadrant photodetector, e.g., is contained in the area $\Omega$. Since the light power of Eq. (20) keeps track of frequency components but integrates over spatial modes and sums over polarization states, one can write an operator equation for the detected light power at frequency $\rho f$ :

$$
P_{\text {det }}(\Omega, \rho)=\frac{\epsilon_{0} c}{2} E_{k l s q}^{*} D_{k l s q, m n r p}(\Omega, \rho) E_{m n r p},
$$

where the sum is taken over all indices. An ideal photodetector does not intermix frequency components, spatial modes, and polarization states, and the operator $D$ can be simplified to

$$
D_{m n r p, k l s q}(\Omega, \rho)=D_{m n, k l}^{M} \otimes D_{r s}^{F \rho} \otimes D_{p q}^{P} .
$$

The operator $D_{m n, k l}^{M}$, which acts on the modal space and accounts for the shape of the detector, is given in Ref. 12 . The $\rho$ th frequency component $\rho f$ is projected out by the operator $D_{r s}^{F \rho}$, which selects the Fourier components $\langle r|$ 
and $|s\rangle$ such that $s-r=\rho$. Polarization states are added in quadrature; therefore

$$
D_{r s}^{F \rho}=\delta_{r, s-\rho}, \quad D_{p q}^{P}=\delta_{p q} .
$$

In a heterodyne detection scheme, additional frequencies are added to the light. In the Pound-Drever-Hall reflection locking technique, ${ }^{14}$ for example, an electro-optic modulator is used to impose phase-modulation sidebands at radio frequencies ( $r f$ 's) on the light incident on a Fabry-Perot cavity. When it is slightly detuned from resonance, the cavity converts the phase modulation to amplitude modulation, and the light reflected from the cavity contains an rf signal that is proportional to the deviation from perfect resonance. There are two approaches to integrating these additional rf frequencies into the above formalism: (1) extending the frequency space or (2) calculating the electric field vectors of the output field separately (assuming that there is no interaction among the different rf components). We use the latter approach, in which case the downconverted power signal can be written as

$$
\begin{aligned}
S(\Omega, \rho)= & \frac{\epsilon_{0} c}{2} E_{\mathrm{CR}}^{*} D(\Omega, \rho) E_{\mathrm{SB}-} \\
& +\frac{\epsilon_{0} c}{2} E_{\mathrm{SB}+}^{*} D(\Omega, \rho) E_{\mathrm{CR}},
\end{aligned}
$$

where $E_{\mathrm{CR}}, E_{\mathrm{SB}+}$ and $E_{\mathrm{SB}-}$ denote the electric field of the carrier, the upper sideband, and the lower sideband, respectively. The above equation is similar to Eq. (21), with the difference that the downconversion selects only the cross terms at the rf frequency.

In general, $S(\Omega, \rho)$ is a complex quantity: The real part describes the in-phase (cosine) term, while the imaginary part describes the quadrature-phase (sine) term of the rf signal. If one adds positive and negative audio frequencies, one obtains a periodic signal at frequency $\rho f$, which describes an ellipse in the complex phase plane:

$$
\begin{aligned}
S & =S(\Omega, \rho)+S(\Omega,-\rho) \\
& =\exp (i \varphi)[a \cos (\rho \omega t+\phi)+i b \sin (\rho \omega t+\phi)],
\end{aligned}
$$

where $a$ and $b$ are the major and minor axes of the ellipse, $\varphi$ gives the orientation of the major axis, and $\phi$ is the phase shift of the signal. In practice, the minor axis often vanishes, i.e., $b=0$, and the signal can be described by its complex transfer function coefficient $a \exp (i \phi)$ and its $\mathrm{rf}$ phase $\varphi$ only.

\section{INPUT BEAM NOISE}

An important consideration in designing an interferometric gravitational-wave detector is the coupling of noise on the input light to the gravitational-wave readout port. The effects of these noise sources can readily be calculated by introducing "noise" operators, which add various noise terms to an otherwise pristine input beam. Important noise sources are the laser frequency noise, the laser amplitude noise, the oscillator phase noise, the oscillator amplitude noise, and the input beam jitter. Oscillator phase and amplitude noise is added to the system by the rf-sideband-generating process, which is done by an rf os- cillator driving a Pockels cell. Angular input beam jitter can be simulated by using the mirror operator from Eq. (14) and applying it to the input beam with a dither amplitude half the angular beam jitter amplitude. ${ }^{5}$ Lateral input beam jitter can be simulated by replacing the modal mirror operator above with the lateral shift operator from Ref. 12.

Since laser and oscillator noise affects neither the modal decomposition nor the polarization state of the input beam, one can write their corresponding noise operators as

$$
N=\mathbf{1}^{M} \otimes N^{F} \otimes \mathbf{1}^{P}
$$

Using the expressions

$$
\begin{gathered}
f(t)=f_{0}+\sum_{\rho \neq 0} \frac{\rho \omega}{2 \pi} \phi_{\rho} \exp (i \rho \omega t), \\
A(t)=A_{0}\left[1+\sum_{\rho \neq 0} \frac{\Delta A_{\rho}}{A_{0}} \exp (i \rho \omega t)\right],
\end{gathered}
$$

for the laser frequency and the laser amplitude, respectively, and following the derivations of Ref. 15, one obtains the laser frequency noise operator $N_{F}^{F}$ and the laser amplitude noise operator $N_{A}^{F}$ :

$$
\begin{aligned}
& \left(N_{F}^{F}\right)_{r s}=\left\langle r\left|\exp \left[-i \sum_{\alpha \beta}|\alpha\rangle\left(T_{F}^{F}\right)_{\alpha \beta}\langle\beta|\right]\right| s\right\rangle, \\
& \left(T_{F}^{F}\right)_{r s}=i \sum_{\rho \neq 0} \phi_{\rho} \delta_{r, s+\rho}, \\
& \left(N_{A}^{F}\right)_{r s}=\mathbf{1}+\sum_{\rho \neq 0} \frac{\Delta A_{\rho}}{A_{0}} \delta_{r, s+\rho} .
\end{aligned}
$$

Representing the rf modulation by a multiplication factor $\exp \left(i \Gamma \cos \omega_{m} t\right)$, with $\Gamma$ as the modulation depth and $\omega_{m}$ as the modulation frequency, one can calculate the oscillator phase noise and the oscillator amplitude noise by making the replacements

$$
\begin{aligned}
\omega_{m} t & \rightarrow \omega_{m} t+\sum_{\rho \neq 0} \varphi_{\rho} \exp (i \rho \omega t), \\
\Gamma & \rightarrow \Gamma_{0}\left[1+\sum_{\rho \neq 0} \frac{\Gamma_{\rho}}{\Gamma_{0}} \exp (i \rho \omega t)\right] .
\end{aligned}
$$

Assuming that $\varphi_{\rho} \ll 1$ and $\Gamma_{\rho} \ll 1$ and again following Ref. 15, one obtains the oscillator noise operator $N_{O}^{F}$, given as

$$
\left(N_{O}^{F}\right)_{r s}=\sum_{\rho \neq 0}\left[\Gamma_{\rho} \ln ^{\prime} J_{x}(\Gamma)+i x \varphi_{\rho}\right] \delta_{r, s+\rho},
$$

where $J_{x}(\Gamma)$ denotes the Bessel function and $x=0,1$, and -1 for the carrier, the upper rf sideband, and the lower rf sideband, respectively. Since oscillator phase noise is also present on the local oscillator of the downconversion, the inverse of the oscillator phase noise operator has to be applied to the output beam before calculating the downconverted signals. Most downconversion circuits, however, implement an amplitude limiter on the local oscillator, so the oscillator amplitude noise operator has to be applied to the input beam only. 


\section{APPLICATION TO COMPLEX OPTICAL SYSTEMS}

Returning to the example of the triangular cavity, we can solve Eq. (1) for the field inside the cavity in the stationary case,

$$
E_{\text {cav }}=\left(\mathbf{1}-G_{\mathrm{rt}}\right)^{-1} T_{1} E_{\text {in }},
$$

and for the cavity transmitted and reflected fields,

$$
\begin{aligned}
& E_{\text {out }}=T_{2} P_{1}\left(\mathbf{1}-G_{\mathrm{rt}}\right)^{-1} T_{1} E_{\mathrm{in}}, \\
& E_{\mathrm{refl}}=\left[T_{1} M_{1}^{-1} G_{\mathrm{rt}}\left(\mathbf{1}-G_{\mathrm{rt}}\right)^{-1} T_{1}+M_{1}^{\dagger}\right] E_{\mathrm{in}} .
\end{aligned}
$$

In practice, one is often interested in calculating the dynamical response of an optical system "on resonance." The length of an optical resonator can be microscopically adjusted so that the power buildup reaches a maximum. If $E_{n}$ is an eigenvector of the round-trip operator $G_{\mathrm{rt}}$ with eigenvalue $e_{n}$, the power buildup for this eigenmode is proportional to

$$
P_{\text {cav }} \propto \frac{1}{\left|1-e_{n} \exp \left(i k_{0} \Delta l\right)\right|^{2}},
$$

where $\Delta l$ is the required length adjustment. The power buildup is maximal for $\Delta l=-\arg \left(e_{n}\right) / k_{0}$. Often one chooses the eigenmode that most closely represents the spatial mode of the incident beam, usually a $\mathrm{TEM}_{00}$ mode [see Eq. (3)].

Figure 2 shows the response of the demodulated signal reflected from a ring cavity as a function of the frequency of the dither applied to the cavity mirror angle. This is referred to as an angular transfer function. Mirror $M_{3}$ is dithered in angle, and the signal is measured by a splitplane photodetector in the near field (close to the input mirror) of the cavity reflection port. At low dithering frequencies, only the horizontal dither is detected in the near field (because of the necessary parity operation in a triangular cavity), whereas the vertical dither is most sensitively measured in the far field (at infinite distance from the input mirror). At higher frequencies both nearand far-field signals are significant and show peaks at the resonance frequencies for the $\mathrm{TEM}_{10}$ and $\mathrm{TEM}_{01}$ modes, respectively. These peaks can be understood as a resonant buildup of either the $\mathrm{TEM}_{10}$ or the $\mathrm{TEM}_{01}$ mode inside the cavity, which happens at the frequencies where the additional phase shift from the free-space propagation cancels the Gouy phase shift. When the power of one of these modes builds up inside the cavity, the amount of light leaking out of the cavity in reflection also increases, which in turn enhances the signal measured by the photodetector. It should be stressed that such a resonant buildup does not invalidate any of the assumptions that went into the formalism. In fact, the model works equally well on resonance and off resonance. The following parameters were used for the calculation: $\lambda$ $=1.064 \mathrm{~nm}$ (laser wavelength), $l_{1}=0 \mathrm{~m}, l_{2}=l_{3}$ $=6 \mathrm{~m}$ (cavity lengths), $\eta=0.6 \pi$ (round-trip Gouy phase shift), $r=0.97$ (amplitude reflectivity coefficient of mirrors $M_{1}$ and $M_{2}$ ), and $f_{\mathrm{rf}}=c / 2 /\left(l_{2}+l_{3}\right)$ (rf modulation frequency). Mirrors $M_{1}$ and $M_{2}$ were flat, whereas mirror $M_{3}$ had a radius of curvature of $\sim 9.2 \mathrm{~m}$, which puts the beam waist right in between $M_{1}$ and $M_{2}$. The input light was a purely vertically polarized $\mathrm{TEM}_{00}$ mode with a cosinusoidal rf modulation. The photodetector at the reflection port was split into two half-planes, which add with opposite sign. The detected output power was down-converted and normalized by the input power, the modulation index, and the amplitude of the dither. The matrix notation of the operators used in the calculations is set out in Appendix A3.

Figure 3 shows a simulation using 50 audio frequency components. The dither signal was an approximated square wave:

$$
\frac{4}{\pi} \sum_{n=1}^{13} \frac{1}{2 n-1} \sin [2 \pi(2 n-1) t] .
$$

Other parameters are the same as those listed above.

Arbitrarily complex configurations such as a system of coupled optical resonators can be simulated by writing down the equivalent equations and using the operators of the previous sections to describe propagation, reflection, and photodetection. This approach is similar to that of

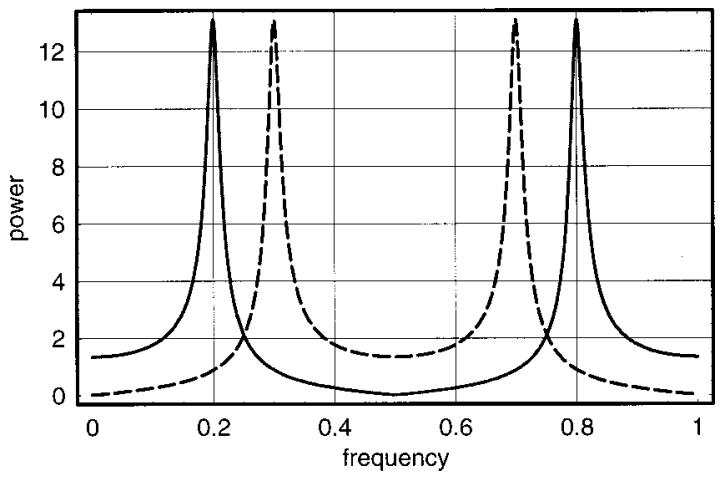

Fig. 2. Angular transfer function of a triangular Fabry-Perot cavity for horizontal (solid curve) and vertical misalignments. Shown is the power of the downconverted signal measured by a split-plane photodetector in the near field of the reflection port as a function of the dither frequency (in units of the free spectral range) of mirror $M_{3}$. For parameters see the text.

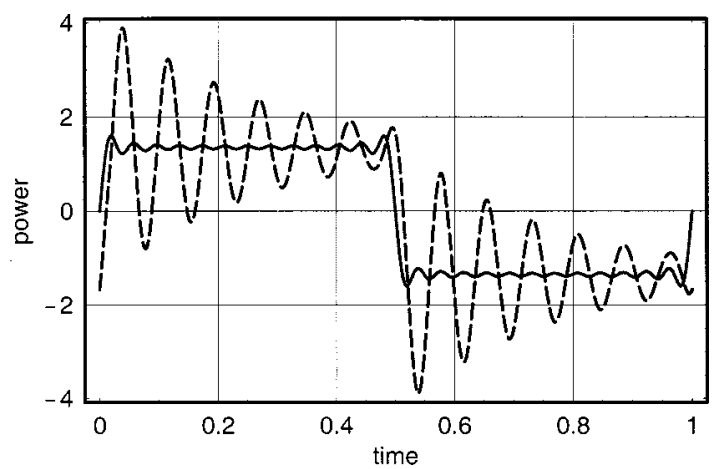

Fig. 3. Response of a triangular cavity to a square-wave dither in angle. Shown is the power of the downconverted signal measured by a split-plane photodetector in the near field of the reflection port while mirror $M_{3}$ is dithered by an approximation of a square-wave signal. The frequency of the dither signal is 1 $\mathrm{kHz}$ (solid curve) and $384.947 \mathrm{kHz}$ (dashed curve). The $1-\mathrm{kHz}$ response is almost identical in shape to the applied dither signal, whereas for the $384.947-\mathrm{kHz}$ dither one of the higher-frequency components of the square wave becomes resonant. See the text for parameters. 


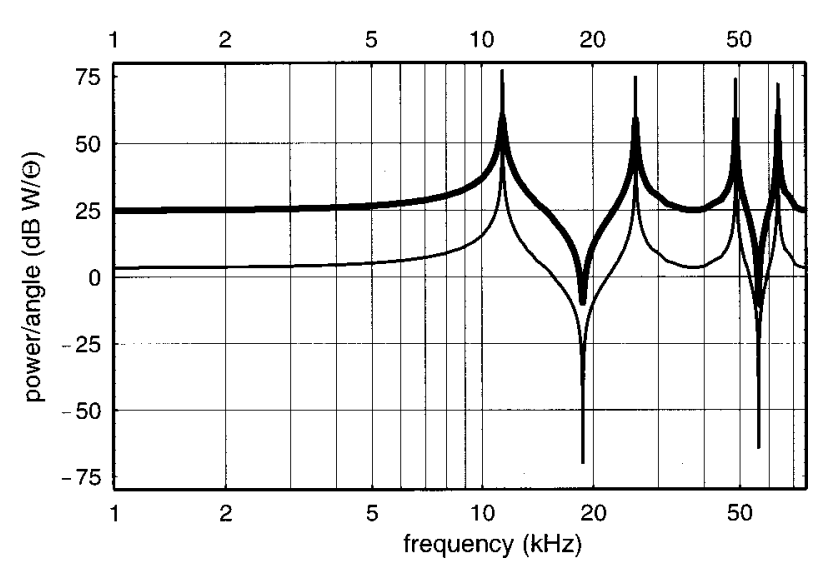

Fig. 4. Response of the LIGO interferometer to a dither of the rear mirror of the in-line arm cavity. Shown is the power of the downconverted signal per unit angle $\left(\Theta \approx 10^{-5} \mathrm{rad}\right)$ measured by a split-plane photodetector in the far field of the antisymmetric port (thick curve) and in the far field of the reflection port (thin curve). The reflection port signal is much narrower, since it experiences a double resonance in both the arm cavity and the power recycling cavity; for higher frequencies the peak values slightly decrease, indicating that the signal is shifted away from the exact resonance in the power recycling cavity. See the text for parameters.

Ref. 12 and was used to build a model of the LIGO interferometer and calculate its angular transfer functions.

Using the equations for the power recycled Michelson interferometer with Fabry-Perot arm cavities in the arms from Ref. 12 and the optical parameters of the LIGO detector, ${ }^{5}$ we calculated the frequency dependence of the angular dithers. Figure 4 shows the alignment signals measured at the antisymmetric port and the reflection port of the interferometer while dithering the end mirror of the in-line arm as a function of the dither frequency. The periodicity of the signal is determined by the free spectral range of the LIGO arm cavity, which is approximately $37 \mathrm{kHz}$. The first resonance in the angular transfer function appears at $11.4 \mathrm{kHz}$, which is the fundamental frequency of the arm cavity transverse modes. The second peak in Fig. 4 is the image of the $11.4-\mathrm{kHz}$ resonance mirrored at the cavity free spectral range. At low frequencies, where the response functions are important for the automatic alignment system, there is no significant frequency dependence.

\section{CONCLUSIONS}

The model described in the present work is capable of simulating a wide set of problems encountered in designing an interferometric gravitational-wave detector: (1) the calculation of displacement and angular sensitivity, (2) the derivation of the frequency dependence of error signals used to lock and align these interferometers, and (3) the study of technical noise sources such as the propagation of laser frequency noise through the system. Compared with methods that are based on fast Fourier transforms ${ }^{16-18}$ or full time-domain simulations, ${ }^{19-21}$ this model is numerically economical because it gives explicit solutions for the laser fields in the interferometer. However, our approach cannot compete with these other methods if the distortions are very large (e.g., small aper- tures, large misalignment angles) or if one is interested in studying transient phenomena. The techniques of this paper were used in the design of the LIGO detector.

In summary, this model is accurate and efficient if the distortions are small, are periodic, and can be expressed as a finite sum of the lowest-order spatial eigenmodes of an optical resonator. It is also versatile in that it allows for building up more complex optical systems with relative simplicity.

\section{APPENDIX A: FORMULAS}

\section{Rotation and Parity Operator}

Going through one round trip of a ring cavity can require a physical rotation of the beam (nonplanar configurations) as well as a parity operation (odd number of mirrors). A physical rotation of the beam will also rotate the polarization state accordingly. However, a rotation of the polarization state can be done independently of a physical rotation of the beam image. The most general rotation of a polarization state can be written as

$$
R^{P}=\exp (-i \mathbf{a} \cdot \boldsymbol{\sigma})=\mathbf{1} \cos |\mathbf{a}|-i \frac{\mathbf{a} \cdot \boldsymbol{\sigma}}{|\mathbf{a}|} \sin |\mathbf{a}|
$$

with $\boldsymbol{\sigma}$ as the Pauli matrices

$$
\boldsymbol{\sigma}_{1}=\left[\begin{array}{ll}
0 & 1 \\
1 & 0
\end{array}\right], \quad \boldsymbol{\sigma}_{2}=\left[\begin{array}{cc}
0 & -i \\
i & 0
\end{array}\right], \quad \boldsymbol{\sigma}_{3}=\left[\begin{array}{cc}
1 & 0 \\
0 & -1
\end{array}\right]
$$

and $\mathbf{a}$ as an arbitrary real vector. Any rotation (including a possible parity operation) can then be written as

$$
R=\left(R^{M} F^{M}\right) \otimes \mathbf{1}^{F} \otimes\left(R^{P} F^{P}\right),
$$

with $F$ as the parity operator that flips (mirrors) the beam along the $y$ axis. In the Hermite-Gaussian basis, we have

$$
\begin{aligned}
F_{m n, k l}^{M} & =(-1)^{m b} \delta_{m k} \delta_{n l}, \\
F^{P} & =\left(-\sigma_{3}\right)^{b}
\end{aligned}
$$

with $b$ as the number of mirror reflections. Starting with a rotation of the electric field

$$
\begin{aligned}
E(x \cos \alpha+y \sin \alpha, y \cos \alpha-x & \sin \alpha) \\
& =R^{M}(\alpha) E(x, y, z),
\end{aligned}
$$

one can expand the left-hand side in a Taylor series and obtain

$$
R^{M}(\alpha)=\exp \left[i \alpha\left(y \frac{1}{i} \frac{\mathrm{d}}{\mathrm{d} x}-x \frac{1}{i} \frac{\mathrm{d}}{\mathrm{d} y}\right)\right]
$$

In the modal basis, this unitary operator can be written as

$$
R_{m n, k l}^{M}(\alpha)=\left\langle m n\left|\exp \left(i \alpha \sum_{o p, q r}|o p\rangle T_{o p, q r}^{\alpha}\langle q r|\right)\right| k l\right\rangle,
$$

with 


$$
\begin{aligned}
T_{o p, q r}^{\alpha}= & \iint_{-\infty}^{\infty} \mathrm{d} x \mathrm{~d} y U_{o}^{\dagger}(x, z) U_{p}^{\dagger}(y, z) \\
& \times\left(\frac{y}{i} \frac{\mathrm{d}}{\mathrm{d} x}-\frac{x}{i} \frac{\mathrm{d}}{\mathrm{d} y}\right) U_{q}(x, z) U_{r}(y, z) .
\end{aligned}
$$

Using the recursion relations for the Hermite-Gaussian polynomials, one can solve the integration:

$$
T_{o p, q r}^{\alpha}=\frac{\sqrt{p q}}{i} \delta_{o, q-1} \delta_{p, r+1}-\frac{\sqrt{o r}}{i} \delta_{o, q+1} \delta_{p, r-1} .
$$

The corresponding rotation of the polarization state is simply Eq. (A1) with $a_{2}=\alpha$ and $a_{1}=a_{3}=0$.

\section{Reflection and Transmission Coefficients}

If a laser beam hits a surface at an angle other than normal, the reflection and transmission coefficients are in general different for perpendicular ( $s$-polarization) and parallel ( $p$-polarization) electric field components. If the $x$ axis is rotated by an angle $\xi$ with respect to the direction of parallel polarization, the amplitude reflection operator $M^{P}$ can be written as

$$
M^{P}=\exp (i \mathbf{a} \cdot \boldsymbol{\sigma})\left[\begin{array}{cc}
r_{p} & 0 \\
0 & r_{s}
\end{array}\right] \exp (-i \mathbf{a} \cdot \boldsymbol{\sigma}),
$$

with $\mathbf{a}=(0, \xi, 0) . \quad$ Similarly, the amplitude transmission operator $T^{P}$ can be deduced from the above equation by replacing the perpendicular and parallel amplitude reflection coefficients, $r_{s}$ and $r_{p}$, with their corresponding amplitude transmission coefficients, $t_{s}$ and $t_{p}$, respectively.

\section{Examples in Matrix Notation}

When calculating the lowest-order perturbations only, one can restrict the modal space to three dimensions $\left(\mathrm{TEM}_{00}, \mathrm{TEM}_{10}\right.$, and $\mathrm{TEM}_{01}$ ) and carry along only three audio frequency components $(0,+f$, and $-f)$. This subection lists the operators in the matrix notation for this finite vector space.

If we use Eq. (12) and relation (13) and assume that propagation length is dithered by $l(t)=l_{0}+\delta l \cos \omega t$, the free-space propagator $P$ becomes

$$
\begin{aligned}
P^{F}(l)= & \exp \left(-i k_{0} l_{0}\right) \\
& \times \exp \left(-i\left[\begin{array}{ccc}
0 & k_{0} \delta l / 2 & k_{0} \delta l / 2 \\
k_{0} \delta l / 2 & \omega l_{0} / c & 0 \\
k_{0} \delta l / 2 & 0 & -\omega l_{0} / c
\end{array}\right]\right), \\
P^{M}(\eta)= & {\left[\begin{array}{ccc}
\exp (i \eta) & 0 & 0 \\
0 & \exp (2 i \eta) & 0 \\
0 & 0 & \exp (2 i \eta)
\end{array}\right], } \\
P^{P}= & {\left[\begin{array}{ll}
1 & 0 \\
0 & 1
\end{array}\right], }
\end{aligned}
$$

neglecting terms of order $\omega \delta l / c$. Similarly, assuming that the mirror orientation is dithered by $\theta(t)$ $=\theta \cos \omega t$, the mirror operators of Eqs. (14)-(17) can be written as

$$
\begin{gathered}
T^{F}=\frac{\Theta}{\sqrt{2}}\left[\begin{array}{ccc}
0 & 1 & 0 \\
1 & 0 & 0 \\
0 & 0 & 0
\end{array}\right], \quad T^{M}=\frac{1}{\sqrt{2}}\left[\begin{array}{ccc}
0 & 1 & 0 \\
1 & 0 & 0 \\
0 & 0 & 0
\end{array}\right], \\
M^{P}=\left[\begin{array}{cc}
r_{p} & 0 \\
0 & r_{s}
\end{array}\right]
\end{gathered}
$$

with $\Theta=\theta \pi w(z) / \lambda$ and with $r_{p}$ and $r_{s}$ as the amplitude reflection coefficients for $p$ and $s$ polarization, respectively. Using the relation of Eq. (6), one can map both the free-space propagator and the mirror operators into an 18-dimensional vector space.

The rotation and parity operators can be expressed as (set $\alpha=0$ for a triangular cavity)

$$
\begin{array}{ll}
R^{M}=\exp \left(i \alpha\left[\begin{array}{ccc}
0 & 0 & 0 \\
0 & 0 & i \\
0 & -i & 0
\end{array}\right]\right), & F^{M}=\left[\begin{array}{ccc}
1 & 0 & 0 \\
0 & -1 & 0 \\
0 & 0 & 1
\end{array}\right], \\
R^{P}=\left[\begin{array}{cc}
\cos \alpha & -\sin \alpha \\
\sin \alpha & \cos \alpha
\end{array}\right], & F^{P}=\left[\begin{array}{cc}
-1 & 0 \\
0 & 1
\end{array}\right] .
\end{array}
$$

The operators for a circular photodetector, $D_{c}$, and a halfplane detector, $D_{h}$ (split along the $y$ axis, segments added with opposite sign), read as

$$
\begin{gathered}
D^{F(+f)}=\left[\begin{array}{lll}
0 & 1 & 0 \\
0 & 0 & 0 \\
1 & 0 & 0
\end{array}\right], \quad D^{F(-f)}=\left[\begin{array}{lll}
0 & 0 & 1 \\
1 & 0 & 0 \\
0 & 0 & 0
\end{array}\right], \\
D^{P}=\left[\begin{array}{ll}
1 & 0 \\
0 & 1
\end{array}\right], \\
D_{c}^{M}=\left[\begin{array}{lll}
1 & 0 & 0 \\
0 & 1 & 0 \\
0 & 0 & 1
\end{array}\right], \quad D_{h}^{M}=\sqrt{\frac{2}{\pi}\left[\begin{array}{lll}
0 & 1 & 0 \\
1 & 0 & 0 \\
0 & 0 & 0
\end{array}\right],}
\end{gathered}
$$

where operators without a subscript are independent of the shape of the detector. Assuming that the laser frequency noise can be expressed by $f(t)=f_{0}$ $+(\omega / 2 \pi) \phi \cos \omega t$, the laser amplitude noise by $A(t)$ $=A_{0}\left(1+\Delta_{A} \cos \omega t\right)$, the oscillator phase noise by $\varphi(t)$ $=\varphi \cos \omega t$, and the oscillator amplitude noise by $\Gamma(t)$ $=\Gamma\left(1+\Delta_{\Gamma} \cos \omega t\right)$, one obtains

$$
\begin{aligned}
N_{F}^{F}= & {\left[\begin{array}{ccc}
1 & -\phi / 2 & \phi / 2 \\
\phi / 2 & 1 & 0 \\
-\phi / 2 & 0 & 1
\end{array}\right], } \\
N_{A}^{F} & =\left[\begin{array}{ccc}
1 & \Delta_{A} / 2 & \Delta_{A} / 2 \\
\Delta_{A} / 2 & 1 & 0 \\
\Delta_{A} / 2 & 0 & 1
\end{array}\right], \\
N_{O \varphi}^{F} & =\left[\begin{array}{ccc}
1 & i x \varphi / 2 & i x \varphi / 2 \\
i x \varphi / 2 & 1 & 0 \\
i x \varphi / 2 & 0 & 1
\end{array}\right],
\end{aligned}
$$




$$
N_{O A}^{F}=\left[\begin{array}{ccc}
1 & |x| \Delta_{\Gamma} / 2 & |x| \Delta_{\Gamma} / 2 \\
|x| \Delta_{\Gamma} / 2 & 1 & 0 \\
|x| \Delta_{\Gamma} / 2 & 0 & 1
\end{array}\right],
$$

with $\Gamma \ll 1$ and $x=0,1$, and -1 for the carrier, the upper rf sideband, and the lower rf sideband, respectively.

For computing the operators for the input beam jitter, one can use the ansatz from Eqs. (16) and (17). If the input beam jitter is parameterized at a distance $z$ from the beam waist position by $\alpha(t)=\alpha \cos \omega t$ (tilt around the $y$ axis) and $\Delta x(t)=\Delta x \cos \omega t$ (displacement along the $x$ axis), the corresponding operators become

$$
\begin{array}{cc}
T_{\alpha}^{F}=\frac{\alpha \pi w(z)}{\sqrt{8} \lambda}\left[\begin{array}{ccc}
0 & 1 & 0 \\
1 & 0 & 0 \\
0 & 0 & 0
\end{array}\right], & T_{\alpha}^{M}=\frac{1}{\sqrt{2}}\left[\begin{array}{ccc}
0 & 1 & 0 \\
1 & 0 & 0 \\
0 & 0 & 0
\end{array}\right], \\
T_{\Delta x}^{F}=-\frac{\Delta x}{\sqrt{8} w(z)}\left[\begin{array}{lll}
0 & 1 & 0 \\
1 & 0 & 0 \\
0 & 0 & 0
\end{array}\right], & T_{\Delta x}^{M}=\frac{1}{\sqrt{2}}\left[\begin{array}{ccc}
0 & t^{*} & 0 \\
t & 0 & 0 \\
0 & 0 & 0
\end{array}\right],
\end{array}
$$

with $t=i-z / z_{0}$.

\section{ACKNOWLEDGMENTS}

We thank our colleagues on the LIGO project, who helped us with many useful suggestions and comments. In particular, we thank P. Fritschel for many clarifying discussions. This work is supported by National Science Foundation cooperative agreement PHY-9210038.

Address correspondence to Daniel Sigg at the location on the title page or by e-mail, sigg_d@ligo.mit.edu.

\section{REFERENCES}

1. A. Abramovici, W. Althouse, J. Camp, J. A. Giaime, A. Gillespie, S. Kawamura, A. Kuhnert, T. Lyons, F. J. Raab, R. L. Savage, Jr., D. Shoemaker, L. Sievers, R. Spero, R. Vogt, R. Weiss, S. Whitcomb, and M. Zucker, "Improved sensitivity in a gravitational wave interferometer and implications for LIGO,” Phys. Lett. A 218, 157-163 (1996).

2. B. Caron, A. Dominjon, C. Drezen, R. Flaminio, X. Grave, F. Marion, L. Massonnet, C. Mehmel, R. Morand, B. Mours, V. Sannibale, M. Yvert, D. Babusci, S. Bellucci, S. Candusso, G. Giordano, G. Matone, J.-M. Mackowski, L. Pinard, F. Barone, E. Calloni, L. DiFiore, M. Flagiello, F. Garuti, A. Grado, M. Longo, M. Lops, S. Marano, L. Milano, S. Solimeno, V. Brisson, F. Cavalier, M. Davier, P. Hello, P. Heusse, P. Mann, Y. Acker, M. Barsuglia, B. Bhawal, F. Bondu, A. Brillet, H. Heitmann, J.-M. Innocent, L. Latrach, C. N. Man, M. PhamTu, E. Tournier, M. Taubmann, J.-Y. Vinet, C. Boccara, P. Gleyzes, V. Loriette, J.-P. Roger, G. Cagnoli, L. Gammaitoni, J. Kovalik, F. Marchesoni, M. Punturo, M. Beccaria, M. Bernardini, E. Bougleux, S. Braccini, C. Bradaschia, G. Cella, A. Ciampa, E. Cuoco, G. Curci, R. DelFabbro, R. DeSalvo, A. DiVirgilio, D. Enard, I. Ferrante, F. Fidecaro, A. Giassi, A. Giazotto, L. Holloway, P. LaPenna, G. Losurdo, S. Mancini, M. Mazzoni, F. Palla, H.-B. Pan, D. Passuello, P. Pelfer, R. Poggiani, R. Stanga,
A. Vicere, Z. Zhang, V. Ferrari, E. Majorana, P. Puppo, P. Rapagnani, and F. Ricci, "The VIRGO interferometer for gravitational wave detection," Nucl. Phys. B54, 167-175 (1997).

3. K. Danzmann, "GEO 600-a 600-m laser interferometric gravitational wave antenna," in First Edoardo Amaldi Conference on Gravitational Wave Experiments, E. Coccia, G. Pizella, and F. Ronga, eds. (World Scientific, Singapore, 1995), pp. 100-111.

4. K. Tsubono, "300-m laser interferometer gravitational wave detector (TAMA300) in Japan," in First Edoardo Amaldi Conference on Gravitational Wave Experiments, E. Coccia, G. Pizella, and F. Ronga, eds. (World Scientific, Singapore, 1995), pp. 112-114.

5. P. Fritschel, G. Gonzlez, N. Mavalvala, D. Shoemaker, D. Sigg, and M. Zucker, "Alignment of a long-baseline gravitational wave interferometer," Appl. Opt. 37, 6734-6747 (1998).

6. J.-Y. Vinet, B. J. Meers, C. N. Man, and A. Brillet, "Optimization of long-baseline optical interferometers for gravitational-wave detection," Phys. Rev. D 38, 433-447 (1988).

7. B. J. Meers, "The frequency response of interferometric gravitational wave detectors," Phys. Lett. A 142, 465-470 (1989).

8. D. Z. Anderson, "Alignment of resonant optical cavities," Appl. Opt. 23, 2944-2949 (1984).

9. N. Sampas and D. Z. Anderson, "Stabilization of laser beam alignment to an optical resonator by heterodyne detection of off-axis modes," Appl. Opt. 29, 394-403 (1990).

10. E. Morrison, B. J. Meers, D. I. Robertson, and H. Ward, "Experimental demonstration of an automatic alignment system for optical interferometers," Appl. Opt. 33, 50375040 (1994).

11. E. Morrison, B. J. Meers, D. I. Robertson, and H. Ward, "Automatic alignment of optical interferometers," Appl. Opt. 33, 5041-5049 (1994).

12. Y. Hefetz, N. Mavalvala, and D. Sigg, "Principles of calculating alignment signals in complex resonant optical interferometers," J. Opt. Soc. Am. B 14, 1597-1605 (1997).

13. A. E. Siegman, Lasers (University Science, Mill Valley, Calif., 1986), Chap. 13, p. 663.

14. R. W. P. Drever, J. L. Hall, F. V. Kowalski, J. Hough, G. M. Ford, A. J. Munley, and H. Ward, "Laser phase and frequency stabilization using an optical resonator," Appl. Phys. B 31, 97-105 (1983).

15. J. B. Camp, H. Yamamoto, S. E. Whitcomb, and D. E McClelland, "Analysis of light noise sources in a recycled Michelson interferometer with Fabry-Perot arms," J. Opt. Soc. Am. A 17, 120-128 (2000).

16. J.-Y. Vinet, P. Hello, C. N. Man, and A. Brillet, "A highaccuracy method for the simulation of non-ideal optical cavities," J. Phys. (Paris) I 2, 1287-1303 (1992).

17. P. Saha, "Fast estimation of transverse fields in highfinesse optical cavities," J. Opt. Soc. Am. A 14, 2195-2202 (1997).

18. B. Bochner, "Modeling the performance of interferometric gravitational-wave detectors with realistically imperfect optics," Ph.D. thesis (Massachusetts Institute of Technology, Cambridge, Mass., 1998).

19. D. Redding and L. Sievers, Jet Propulsion Laboratory, 4800 Oak Grove Drive, Pasadena, California 91109 (personal communication, August 1997)

20. B. Bhawal, "Real-time simulation of interferometric gravitational-wave detectors involving moving mirrors," J. Opt. Soc. Am. A 15, 120-143 (1998).

21. R. G. Beausoleil and D. Sigg, "Spatiotemporal model of the LIGO interferometer," J. Opt. Soc. Am. A 16, 2990-3002 (1999). 